Luxembourg

\section{Finance and trade}

Consumer price index $(1970=100) 196078,1976155.6$; growth 1960-70 $2.5 \% \mathrm{pa}, 1970-767.6 \% \mathrm{pa}$

Money stock The main part of money stock used is Belgian currency which is also legal tender; Luxembourg notes and coins (1977): L Fr $600 \mathrm{mn}$

Budget (1977) Revenue: L Fr $33406 \mathrm{mn}=\$ 932 \mathrm{mn}=£ 534 \mathrm{mn}$

Expenditure: $\mathrm{L}$ Fr $34338 \mathrm{mn}=\$ 958 \mathrm{mn}=£ 549 \mathrm{mn}$

Balance of payments see Belgium

International reserves See Belgium; of total reserves held by

Belgium-Luxembourg, Luxembourg held (1976) \$19 mn in SDRs and

reserve position in the International Monetary Fund

External trade (1975) ${ }^{\mathrm{a}}$

Imports (fob) ${ }^{\mathrm{b}}: \mathrm{L}$ Fr $73460 \mathrm{mn}=\$ 1997 \mathrm{mn}=£ 899 \mathrm{mn}$

Exports $^{\mathrm{b}}$ : L Fr $67750 \mathrm{mn}=\$ 1842 \mathrm{mn}=£ 829 \mathrm{mn}$

Main export Steel $90^{*} \%$

aIncluded with Belgium in the Belgium-Luxembourg customs union; see Belgium for union products and direction 'Including services

\section{Special focus}

Steel prices (producers basic price, West Germany)

\begin{tabular}{|c|c|c|c|c|c|}
\hline & $\begin{array}{l}\text { \$per } \\
\text { tonne }\end{array}$ & $\begin{array}{l}\% \text { change over } \\
\text { previous year }\end{array}$ & & $\begin{array}{l}\$ \text { per } \\
\text { tonne }\end{array}$ & $\begin{array}{l}\% \text { change over } \\
\text { previous year }\end{array}$ \\
\hline 1970 & 125 & +25 & 1974 & 249 & +21 \\
\hline 1971 & 137 & +10 & 1975 & 300 & +20 \\
\hline 1972 & 157 & +15 & 1976 & 329 & +10 \\
\hline 1973 & 206 & +31 & 1977 & 321 & -2 \\
\hline
\end{tabular}

\section{Malta \\ Republic of Malta \\ Ir-Republika ta’ Malta}

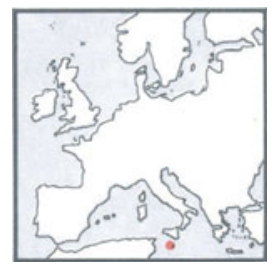

Location Mediterranean Sea

About halfway between Gibraltar and Port Said, with Sicily $93 \mathrm{~km}$ to the north and Tunisia $300 \mathrm{~km}$ to the west. The islands include Malta, Gozo and Comino Land Area $316 \mathrm{~km}^{2}=122 \mathrm{mi}^{2}$, of which, Malta $245 \mathrm{~km}^{2}$, Gozo $67 \mathrm{~km}^{2}$ Climate Mediterranean (hot summers and warm winters)

Weather at Valletta, 70 maltitude

Temperature: hottest month Aug $23-29^{\circ} \mathrm{C}$, coldest Jan $10-14{ }^{\circ} \mathrm{C}$ Rainfall (av monthly) : driest month July $0 \mathrm{~mm}$, wettest Dec $110 \mathrm{~mm}$ Time 1 hour ahead of GMT (summer time, 2 hours ahead)

Measures Metric and UK (imperial) systems, converting to a completely metric system; local measures are also in use, including:

length 1 palmi $=10.29$ inches $=26.13$ centimetres

weight (mass) 1 rotolo or ratal $=0.794$ kilogram $=1.75$ pounds

1 wizna $=5$ rotoli $=3.969$ kilograms $=8.75$ pounds

1 qantar $=20$ wizna $=100$ rotoli $=79.38$ kilograms $=175$ pounds

Monetary unit Maltese pound $(\mathrm{M} £)=100$ cents $=1000$ mils

Rate of exchange $(1976 \mathrm{av})$ : free $\$ 2.353=\mathrm{M} £ 1, £ 1.303=\mathrm{M} £ 1$

\section{Summary}

Political Republic, which became independent from the United Kingdom on September 21, 1964. The United Kingdom use of a defence base on the island is planned to end in 1979. Member of UN, Council of Europe and Commonwealth

Economic Tourism and light manufacturing industry have been developed to replace a gradual loss of income from UK defence expenditure.

Receipts from tourism equal nearly one-third of exports; main

domestic products exported are clothing and textiles. Ship-repairing is important

\section{People, resources and equipment}

Population 1960329 000, $1970326000,1976330000 *$

Growth: $1960-70-0.1 \% \mathrm{pa}, 1970-760.2 * \% \mathrm{pa}$

Density (1976): $1040 *$ people per $\mathrm{km}^{2}$

Vital statistics (rate per 1000 people, 1976) : births 18.0, deaths 9.0

Regions (Maltese population in 000, 1976; total of 305000 )

Malta 283, Gozo and Comino 22

Towns (population in 000, 1976) Malta Valletta (capital) 14, Sliema 20*,

Birkirkara 17*, Qormi 15* Gozo Victoria (or Rabat) 5

Race (1961) Maltese $96 \%$, English $2 \%$, Italian $1 \frac{1}{2} \%$

Language Maltese and English
Religion (1970) Roman Catholic 98** \%

Education (1975/76) Pupils 64 387, teachers 4169

Labour force (1975) 112 596; in manufacturing 31287 (28\%)

Personnel (1975) Physicians: 382, 1 per 850 people

Standard of living

National income per person (1976): $\mathrm{M} £ 652=\$ 1533=£ 849$

Consumption per person (1975): energy $1032 \mathrm{~kg}$ coal equivalent,

electricity (production) $1020 * \mathrm{~kW} \mathrm{~h}$, newsprint $1.2 \mathrm{~kg}$

Newspapers (1974): number 6

Telephones (Dec 1976): 62 324, 188 per 1000 people

Livestock $(000,1976)$ Cattle $11^{*}$, pigs $25^{*}$, goats $11^{*}$, chickens $1170^{*}$

Electrical capacity (1975) 115 megawatts

Hospital beds (1971) 3431,1 per 95 people

Roads (1974) $1267 \mathrm{~km}=787 \mathrm{mi}$, density $4.0 \mathrm{~km}$ per $\mathrm{km}^{2}$

Ships (registered, 1977) 44, total of 100420 gross tons

Port Valletta

Airport Luqa (Valletta): passenger departures and arrivals (1976) 746000

Durable equipment

(at end-year)

Radio sets (1973)

Television sets (1976)

Passenger cars (1976)

Commercial vehicles (1976)

000 no per

1000 people

129401

no per

$\begin{array}{ll}53 & 160\end{array}$

$13 \quad 40$

$\mathrm{km}$ of road

42

10

\section{Production}

Gross domestic product 1976: $\mathrm{M} £ 203.7 \mathrm{mn}=\$ 479 \mathrm{mn}=£ 265 \mathrm{mn}$ Growth in real terms: $1960-704.9 \% \mathrm{pa}, 1970-768.6 * \% \mathrm{pa}$

Agricultural production index $(1970=100) 196063,197695$;

growth: $1960-704.8 \% \mathrm{pa}, 1970-76-0.8 \% \mathrm{pa}$

Main products Agriculture $(000 \mathrm{t}, 1976)$ Potatoes 20*, wheat 3*,

tomatoes $17^{*}$, cauliflowers $4^{*}$, onions $4^{*}$, grapes $4^{*}$, wine $2^{*}$, milk $25^{*}$,

pigmeat $4^{*}$, poultry meat $3 *$, fish catch 2 Other (1975) Manufactured gas $5 \mathrm{mn} \mathrm{m}{ }^{3}$, electricity (1975/76; year ending March $\left.31 \mathrm{st}\right) 338 \mathrm{mn} \mathrm{kW} \mathrm{h}$, cigarettes $740 \mathrm{mn}$ units, salt 2

\begin{tabular}{|c|c|c|c|c|c|}
\hline $\begin{array}{l}\text { Transport traffic } \\
\text { Air (mn) }\end{array}$ & 1960 & 1970 & 1976 & \multicolumn{2}{|c|}{$\begin{array}{l}\text { Growth \%pa } \\
1960-70 \quad 1970-76\end{array}$} \\
\hline Passenger-kilometres & - & 202 & 340 & na & 9.1 \\
\hline Cargo: tonne-kilometres & - & 2.7 & 3.8 & na & 5.9 \\
\hline Sea: tonnes $(\mathrm{mn})$ & & & & & \\
\hline Goods loaded & 0.040 & 0.050 & 0.133 & 2.2 & 17.7 \\
\hline unloaded & 0.443 & 0.956 & 1.000 & 8.0 & 0.8 \\
\hline & & & & & \\
\hline Number of visitors & 20 & 170 & 340 & 24.1 & 12.2 \\
\hline Gross receipts $(\$ \mathrm{mn})$ & na & 24 & 67 & na & 18.7 \\
\hline
\end{tabular}

\section{Finance and trade}

\begin{tabular}{|c|c|c|c|c|c|}
\hline $\begin{array}{l}\text { Price index } \\
(1970=100)\end{array}$ & 1960 & 1970 & 1976 & $\begin{array}{l}\text { Growth } \\
1960-70\end{array}$ & $\begin{array}{l}\% \text { pa } \\
1970-76\end{array}$ \\
\hline Consumer prices & 84.0 & 100.0 & 133.7 & 1.8 & 5.0 \\
\hline $\begin{array}{l}\text { Money stock } \\
\text { (end-year, } \mathbf{M} £ \mathrm{mn} \text { ) }\end{array}$ & 27.6 & 58.6 & 144.6 & 7.8 & 16.2 \\
\hline
\end{tabular}

$\begin{array}{llllll}\text { (end-year, } \mathrm{M} £ \mathrm{mn} \text { ) } & 27.6 & 58.6 & 144.6 & 7.8 & 16.2\end{array}$

Budget (total, 1976/77; year ending March 31st)

Revenue: $M £ 100.8 \mathrm{mn}=\$ 234 \mathrm{mn}=£ 135 \mathrm{mn}$,

of which, rent for defence facilities $M £ 13.0 \mathrm{mn}=\$ 30 \mathrm{mn}=£ 17 \mathrm{mn}$

Expenditure: $\mathrm{M} £ 96.1 \mathrm{mn}=\$ 223 \mathrm{mn}=£ 129 \mathrm{mn}$

$\begin{array}{llll}\text { Balance of payments }(\$ \mathrm{mn}) & 1972 & 1973 & 1974\end{array}$

$\begin{array}{llllll}\text { Balance of goods (fob) } & -95 & -105 & -162 & -158 & -140\end{array}$

Balance of services $\quad+88+109+130+181+129$

Balance of transfers $\quad+32+32+44+41+75$

\begin{tabular}{llllll} 
Current balance & +25 & +36 & +43 & +41 & +75 \\
\hline & +27 & +63
\end{tabular}

Long-term capital flow $\quad+27 \quad-12+17+32+35$

$\begin{array}{lrrrrr}\text { Reserves and debt (end-year, } \$ \text { mn) } & & & & \\ \text { International reserves } & 275 & 325 & 402 & 500 & 622\end{array}$

$\begin{array}{lrrrrr}\text { External public debt } & 24 & 28 & 36 & 48 & 58\end{array}$

External trade (1976) Imports: $M £ 180 \mathrm{mn}=\$ 423 \mathrm{mn}=£ 234 \mathrm{mn}$

Exports: $\mathrm{M} £ 97 \mathrm{mn}=\$ 229 \mathrm{mn}=£ 127 \mathrm{mn}$

Main imports $\%$ of total Main exports $\%$ of total

Food $\quad 20 \quad$ Clothing

(of which, cereals 6) Machinery

Textile yarns and fabrics $16 \quad$ Petroleum products

Petroleum products 9

Machinery, non-electric 8

Chemicals

Food

Electrical machinery $\quad 6$

Main sources

United Kingdom

Food 6

Textile yarns and fabrics 5

Ships and boats 5

Tobacco

Italy

West Germany

United States

France

24

Main destinations

West Germany

United Kingdom

Libya

Italy

Netherlands 